\title{
Measurements Analysis of the Software-Related Power Consumption in Microprocessors
}

\author{
N. Kavvadias, P. Neofotistos, S. Nikolaidis, K. Kosmatopoulos and Th. Laopoulos \\ Electronics Lab. Physics Dept., Aristotle University of Thessaloniki, \\ Thessaloniki, 54124, Greece, \\ Email: laopoulos@physics.auth.gr
}

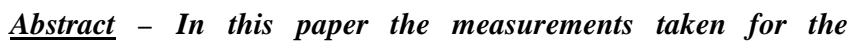
development of instruction-level energy models for microprocessors are presented and analyzed. An appropriate measuring environment and a suitable measuring methodology was developed for taking the necessary measurements. The energy of an instruction is defined as a sum of three components. The pure base energy cost, the inter-instruction cost and the effect of the energy sensitive factors (instruction parameters). These components are characterized for each instruction of the ARMTTDMI embedded processor and their values are analyzed. Using the resulted models estimates of the energy consumption of real software kernels with only up to $5 \%$ error was determined.
\end{abstract}

\section{INTRODUCTION}

Last years, low power consumption has been established as the third main design target for digital systems together with performance and area. Consequently, accurate estimators of the power consumption at the system as well as the lower level of abstraction are necessary. A large number of embedded computing applications are power or energy critical. Early work on processor analysis had focused on performance improvement without determining the powerperformance tradeoffs. Recently, significant research in low power design and power estimation and analysis has been developed.

Embedded software power modeling techniques are distinguished into two main categories, physical measurement-based and simulation-based ones. In measurement-based approaches [1-7], the energy consumption of software is characterized by data obtained from real hardware. The advantage of measurement-based approaches is that the resulting energy model proves close to the actual energy behavior of the processor.

In measurement techniques, a common practice is to associate instructions running on the processor with their corresponding energy cost. Accurate instruction-level power or energy models can be created by monitoring the instantaneous current drawn by the processor and then calculating the energy of the instruction. For this purpose a measuring environment has been proposed by the authors in [8] using a high performance current mirror based on BJT transistors as current sensing circuit. Also an instruction-level energy consumption modeling methodology has been proposed [9] aiming in the creation of highly accurate models. In this paper the results of our experiments are presented and analyzed.

\section{INSTRUCTION-LEVEL ENERGY MODELING}

The energy consumed during the execution of instructions can be distinguished according to [1] in two components. The base cost, energy amount needed for the execution of the operations which are imposed by the instructions, and the inter-instruction cost which corresponds to an energy overhead due to the changes in the state of the processor provoked by the successive execution of different instructions. Measurements for determining these two energy amounts for each instruction of the ARM7TDMI processor were taken and presented in [10]. However the base costs in [10] were for specific operand and address values (zero operand and immediate values and specific address values to minimize the effect of 1s). This base cost is called pure base cost.

We have observed in our measurements that there is also a dependency of the energy consumption of the instructions on the values of their parameters (register number, operand values, operand addresses, etc). To create accurate models this dependency has to be determined. Additional measurements were taken to satisfy this necessity. It was observed by the measurements that there is a close to linear dependency of the energy on these parameters, versus the number of $1 \mathrm{~s}$ in their word space. Consequently, the effect of any of the above energy-sensitive factors can be efficiently modeled by a coefficient.

Making some appropriate experiments we observed that the effect of each energy-sensitive factor on the energy cost of the instruction is independent of the effect of the other factors. The effects on the energy of these factors are uncorrelated as can be observed in Table 1. As opval, we denote the operand values energy-sensitive factor, whereas regnum corresponds to the register number factor. The distortion of our results from this conclusion is, most of the time, less than $2-3 \%$ and only in some marginal cases becomes more than $7 \%$. According to this conclusion, the effect of the energy-sensitive factors can simply be added to give the total energy amount.

Other sources of energy consumption are conditions of the processor, which lead to an overhead in clock cycles because of the appearance of idle cycles. This, for example, is the case 
of the appearance of pipeline stalls. The effect of such cases on the energy consumption was measured.

Table I. Comparison of measured to calculated instruction energy costs (nJoules) due to additional dependencies

\begin{tabular}{|c|c|c|c|c|c|}
\hline & \multicolumn{2}{|c|}{ Measured } & \multicolumn{2}{|c|}{ Calculated } & \\
\hline Instruction formation & opval & \begin{tabular}{|l|} 
opval+ \\
regnum
\end{tabular} & opval & \begin{tabular}{|l|} 
opval+ \\
regnum
\end{tabular} & $\% \mathrm{~d}$ \\
\hline ADD $R_{d}, R_{n}, R_{s}$, ASR $R_{m}$ & 2.58 & 2.61 & 2.57 & 2.61 & -0.0 \\
\hline $\begin{array}{l}\text { ADD R } R_{d}, R_{n}, R_{s}, A S R \\
\text { \#imm }\end{array}$ & 1.67 & 1.60 & 1.55 & 1.60 & -0.2 \\
\hline ADD $R_{d}, R_{n}, R_{s}$ & 1.51 & 1.59 & 1.51 & 1.56 & 1.7 \\
\hline $\mathrm{ADD} \mathrm{R}_{\mathrm{d}}, \mathrm{R}_{\mathrm{n}}, \mathrm{R}_{\mathrm{s}}, \mathrm{RRX}$ & 1.51 & 1.63 & 1.52 & 1.64 & -1.1 \\
\hline $\mathrm{LDR} \mathrm{R}_{\mathrm{d}},\left[\mathrm{R}_{\mathrm{n}}, \mathrm{R}_{\mathrm{s}}\right]$ & 3.07 & 3.27 & 2.97 & 3.29 & -0.6 \\
\hline STR $R_{d},\left[R_{n}, R_{s}\right]$ & 2.28 & 2.48 & 2.23 & 2.43 & 2.0 \\
\hline
\end{tabular}

\section{PURE BASE COST AND INTER-INSTRUCTION COST MODELS - ANALYSIS OF THE RESULTS}

The complete models for the instruction-level energy consumption of the ARM7TDMI can be found in [11]. Thousand experiments corresponding to the execution of loops of instruction instances on the processor to realize the appropriate pipeline conditions [9] were implemented. For the measurement of the instantaneous current of the processor the measuring environment proposed in [8] was employed. Pure base costs of all the instructions and for all the addressing modes are given. Since the number of the possible instruction pairs (taking into account the addressing modes) is enormous, groups of instructions and groups of addressing modes according to the resources they utilize, have been formed and inter-instruction costs have been given only for representatives of these groups. In this way we keep the size of the required model values reasonable without significant degradation of the accuracy (less than 5\% in the interinstruction cost by using only representative instructions).

For measuring the pure base costs, loops with a reference instruction (we have selected the NOP) and the one test instruction were executed. The energy of the test instruction was calculated as the sum of the energy consumed in the clock cycles required for this instruction to be executed minus two times the energy budget of the NOP instruction. (Due to the pipeline structure, two NOP instructions are also executed in the clock cycles needed for the execution of a test instruction).

In the measurements for the inter-instruction costs, program loops featuring appropriate instruction pairs were formed. In this case, in four clock cycles one Instr1, one Instr 2 and two reference instructions are executed.

Some results for the pure base cost are shown in Table 2 . The values of the pure base costs present most of the time a difference less than $20 \%$ in the energy of the instructions which are executed in the same number of cycles. Also, it has been shown that the existence of a condition in the instruction does not influence significantly the energy of the instruction.
For the instructions which need more cycles (clocks per instruction more than 1, CPI>1) the energy cost increases according to the required cycles. In this way, a computationally efficient model with less but not unacceptable accuracy (depending on the aim of its use) can be provided by assigning energy values to the instructions only according to the number of cycles they need.

Instructions that provably belong to the same instruction groups, are considered to exhibit similar variations to the base energy cost due to effects that could be modeled as interinstruction related. Since instructions belonging to the same instruction group are using the same hardware resources it is probable that the inter-instruction effect between them will be in comparison small, the opposite goes when the instruction utilize different system resources.

Table II. Base cost energy consumption (for zero operands)

\begin{tabular}{|l|r|}
\hline Instruction & $\mathrm{E}(\mathrm{nJ})$ \\
\hline ADD R2, R0, R1 & 0.910 \\
\hline AND R2, R0, R1 & 0.856 \\
\hline ORR R2, R0, R1 & 0.907 \\
\hline ORRS R2, R0, R1 & 0.967 \\
\hline MOV R2, R1 & 0.935 \\
\hline MOV R0, R0 & 0.903 \\
\hline ADD R2, R0, R1, ASR R3 & 2.137 \\
\hline B label & 3.095 \\
\hline LDR R2, [R1, R3] & 2.774 \\
\hline STR R2, [R1, R3] & 1.961 \\
\hline MUL R2, R0, R1, R10 & 2.768 \\
\hline MLA R2, R0, R1, R10 & 3.748 \\
\hline CMP R0, R1 & 0.751 \\
\hline SWP R2, R0, [R1] & 3.917 \\
\hline MRS R2, CPSR & 0.977 \\
\hline MSR CPSR_f, R2 & 1.143 \\
\hline
\end{tabular}

For example, when executing data-processing instructions, the second (flexible) operand determines which functional units shall be used for executing the instruction. Thus, we can distinguish 4 different categories for the data-processing instructions, which are shown in Table 3 . The corresponding inter-instruction costs are given in Table 4. This concept is also applied to other instruction types, so that only a representative part of the instruction and addressing mode range has to be explicitly measured.

Table III. Grouping of the data-processing instruction addressing modes based on architectural characteristics

\begin{tabular}{|c|l|l|}
\hline Group & $2^{\text {nd }}$ source operand functionality & $\begin{array}{c}\text { Addressing } \\
\text { modes }\end{array}$ \\
\hline 1 & Shift amount from a register & $1,3,5,7$ \\
\hline 2 & Shift amount from immediate & $2,4,6,8,11$ \\
\hline 3 & Register operand & 9 \\
\hline 4 & Immediate operand & 10 \\
\hline
\end{tabular}


Most of the values of the inter-instruction costs have negative sign as it was expected due to the followed approach. The contribution of the inter-instruction costs remains small. As it can be observed by our models most of the inter-instruction costs are less than 5\% of the corresponding pure base costs while almost all the cases are covered by an $15 \%$ percentage.

Table IV. Inter-instruction effect costs for representative addressing modes of the ADD instruction

\begin{tabular}{|c|r|r|r|r|}
\hline $\begin{array}{c}\text { Addressing } \\
\text { mode } \\
\text { group }\end{array}$ & 1 & \multicolumn{1}{c|}{2} & \multicolumn{1}{c|}{3} & \multicolumn{1}{c|}{4} \\
\hline 1 & -0.332 & -0.215 & -0.232 & -0.159 \\
\hline 2 & -0.269 & -0.177 & -0.165 & -0.103 \\
\hline 3 & $-9.02 \mathrm{E}-02$ & $-5.98 \mathrm{E}-02$ & -0.186 & -0.2 \\
\hline 4 & -0.141 & $-5.35 \mathrm{E}-02$ & $-9.08 \mathrm{E}-02$ & $-7.53 \mathrm{E}-02$ \\
\hline
\end{tabular}

To determine the accuracy of the method a number of programs with various instructions $(\mathrm{CPI}=1$ or $\mathrm{CPI}>1)$ have been created [10]. In these instructions the operand values were kept zero and thus the effect of energy sensitive factors wasn't taken into account. The energy consumed during the execution of each program was calculated directly from measurements and also calculated by using the instructionlevel energy models derived by the proposed method. The error was found to be up to $1.5 \%$.

According to the above, the energy, $E_{i}$, consumed during the execution of the $i$ instruction can be modeled as:

$$
E_{i}=b_{i}+\sum_{i} a_{i, j} N_{i, j}
$$

where $b_{i}$ is the pure base cost of the $i$ instruction, $a_{i, j}$ and $N_{i, j}$ is the coefficient and the number of $1 \mathrm{~s}$ of the $j$ energy-sensitive factor of the $i$ instruction, respectively. The coefficients for the energy-sensitive factors are given in Table 5.

Each instruction may contain some of these energysensitive factors. The effect of all these factors have to be taken into account to create the energy budget of the instruction.

Table V. Energy-sensitive factor coefficients

\begin{tabular}{|l|c|}
\hline \multicolumn{1}{|c|}{ Energy-sensitive factor } & Coefficient \\
\hline \hline Register number & $a_{i, 1}$ \\
\hline Register value & $a_{i, 2}$ \\
\hline Immediate value & $a_{i, 3}$ \\
\hline Operand value & $a_{i, 4}$ \\
\hline Operand address & $a_{i, 5}$ \\
\hline Fetch address & $a_{i, 6}$ \\
\hline
\end{tabular}

Having modeled the energy cost of the instructions, the energy consumed for running a program of $n$ instructions can be estimated as:

$$
P E=\sum_{1}^{n} E_{i}+\sum_{1}^{n-1} O_{i, i+1}+\sum \varepsilon
$$

where $O_{I, j}$ is the inter-instruction cost of the instructions $i$ and $j$, and $\varepsilon$ is the cost of a pipeline stall.

\section{ANALYSIS OF THE ENERGY DEPENDENCY ON THE INSTRUCTION PARAMETERS}

The dependency of the energy of the instructions on the values of the instruction parameters and the operands, called energy sensitive factors, was also studied. Energy depends on the number of $1 \mathrm{~s}$ in the word structures of these entities. The energy-sensitive factors are the register numbers, the register values, the immediate values, the operand values, the operand addresses and the fetch addresses of the instructions. The effect of each factor was studied separately from the others since the correlation between the effect of these factors is insignificant (see Table 1).

The observed energy dependency can be approximately with sufficient accuracy by linear functions. Coefficients should be derived for each instruction for any energy sensitive factor. However, appropriate grouping of the instructions is used to keep reasonable the number of required coefficients to increase the applicability of the method without significant loss in the accuracy.

The grouping of the instructions for the derivation of the coefficients and the corresponding measurements are presented in [11]. According to the results the linear dependency mentioned above is obvious. Some results are presented here. In Figure 1 the effect of the register number for data-processing instructions in register addressing mode is presented. The actual physical measurements versus estimated energy values for the ADC instruction is shown in Table 6 where the achieved for the selected coefficient accuracy is also given. The error is less than $3 \%$. Such a value of the error characterizes all the selected coefficients.

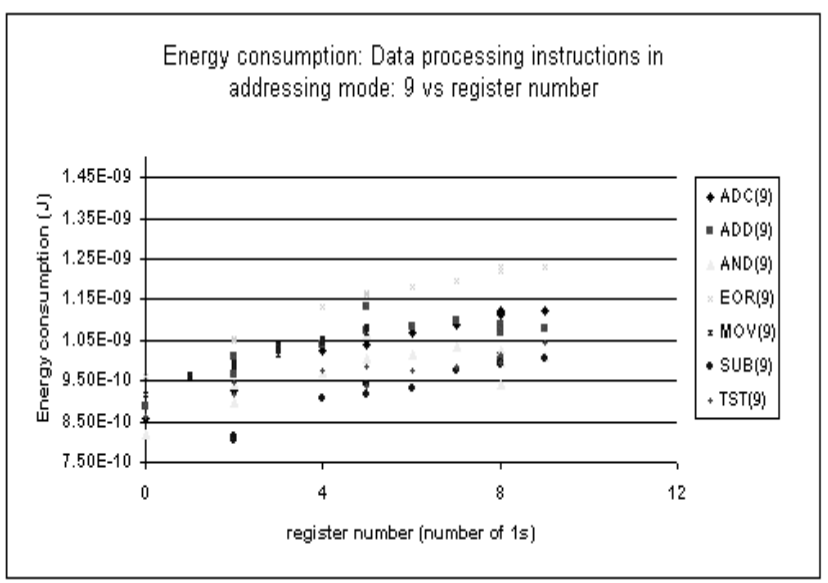

Fig. 1. The effect of register number for data-processing instructions in register addressing mode 
Table VI. Actual physical measurements against estimated energy values for the $\mathrm{ADC}(9)$

\begin{tabular}{|c|c|c|c|c|c|}
\hline Num 1s & Estimated & Measured & $\%$ error & \multicolumn{2}{|c|}{ Model parameters } \\
\hline 0 & 0.874 & 0.855 & 2.20 & & \\
\hline 2 & 0.936 & 0.929 & 0.74 & & \\
\hline 2 & 0.936 & 0.924 & 1.23 & & \\
\hline 5 & 1.028 & 1.040 & 1.19 & & \\
\hline 6 & 1.059 & 1.067 & 0.77 & & \\
\hline 8 & 1.121 & 1.119 & 0.16 & $\mathrm{a}_{\mathrm{i}, 1}$ & $3.08 \mathrm{E}-02$ \\
\hline 8 & 1.121 & 1.124 & 0.28 & $\mathrm{~b}$ & 0.874 \\
\hline 9 & 1.151 & 1.124 & 2.47 & & \\
\hline 7 & 1.090 & 1.088 & 0.17 & & \\
\hline 5 & 1.028 & 1.054 & 2.46 & & \\
\hline 8 & 1.121 & 1.114 & 0.61 & & \\
\hline 4 & 0.997 & 1.023 & 2.51 & & \\
\hline
\end{tabular}

The energy consumption versus the aggregate number of $1 \mathrm{~s}$ in the register values for the ADD instruction in the register addressing mode is given in Figure 2. The linear dependency is obvious.

The quantities which are transferred from memory to register or inversely during the execution of load or store instructions also affect the energy of the instructions. The energy consumption versus the number of $1 \mathrm{~s}$ in operand values for the STR instruction is shown in Figure 3. The effect of operand addresses in the energy consumption of load and store instructions versus the number of $1 \mathrm{~s}$ is evaluated by varying the offset operand values which corresponds to the displacement on a specified base memory address.

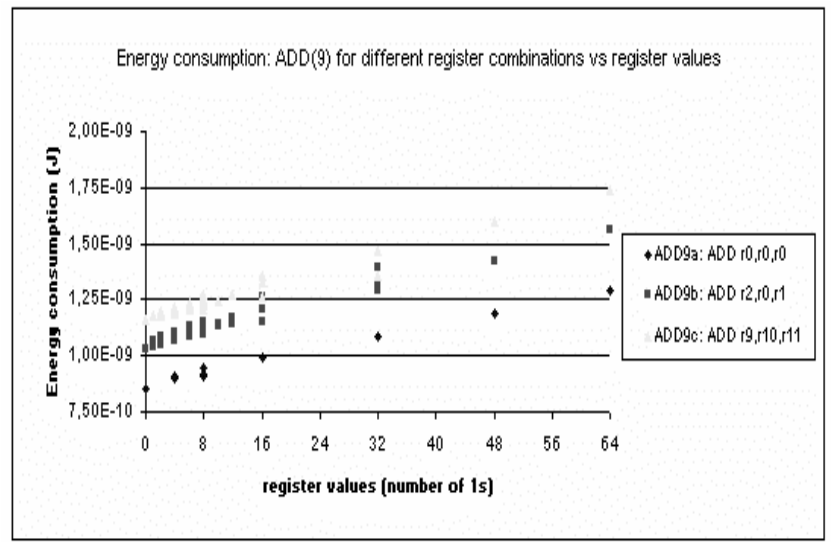

Fig. 2. Energy consumption versus the aggregate number of $1 \mathrm{~s}$ in the register values for the $A D D$ instruction in register addressing mode

Test measurements are given in Figure 4 and the extracted coefficients for the effect of the operand values are given in Table 7. As can be seen from the coefficient values in [11] all the sensitive-energy factors except the register values present almost equivalent effect on the energy of the instruction. The effect of the register values is one order lower except of the case of multiplications where it has similar to the others energy-sensitive factors effect.

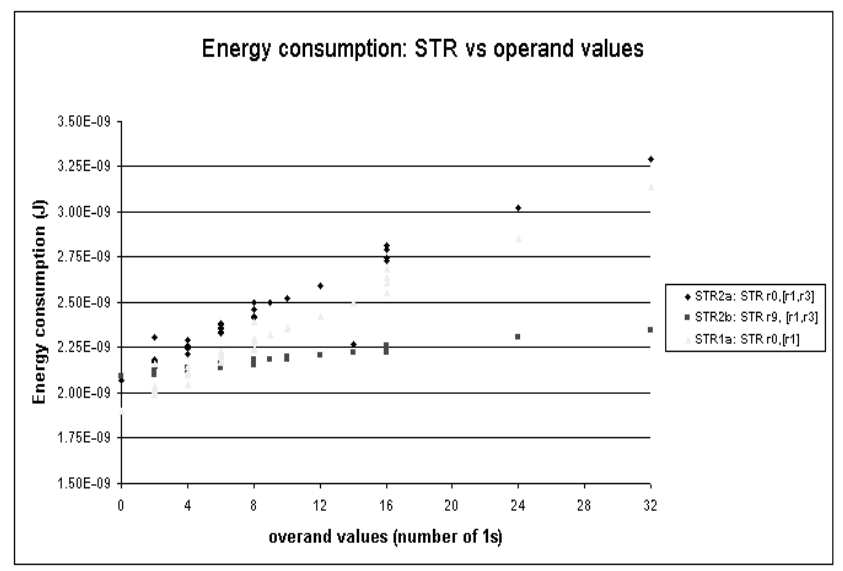

Fig. 3. Energy consumption versus the number of $1 \mathrm{~s}$ in operand values for the STR instruction

Finally there is only a moderate dependence versus the number of $1 \mathrm{~s}$ in the instruction fetch address and therefore the corresponding energy cost is not considered in our models.

Table VII. Coefficients $\left(a_{i, 5}\right)$ for the operand addresses effect on load and store instructions

\begin{tabular}{|c|c|c|c|}
\hline $\begin{array}{l}\text { Instruction } \\
\text { type }\end{array}$ & $\begin{array}{l}\text { Addressing mode } \\
\text { group }\end{array}$ & $\begin{array}{l}\text { Coefficient } \\
\text { value }\end{array}$ & Std. Dev. \\
\hline LDR & Immediate offset & $4.55 \mathrm{E}-02$ & \multirow{3}{*}{$2.41 \mathrm{E}-03$} \\
\hline LDR & Register offset & $2.58 \mathrm{E}-02$ & \\
\hline LDR & $\begin{array}{l}\text { Scaled register } \\
\text { offset }\end{array}$ & $5.20 \mathrm{E}-03$ & \\
\hline STR & Immediate offset & $3.85 \mathrm{E}-02$ & \multirow{3}{*}{ 8.31E-04 } \\
\hline STR & Register offset & $1.61 \mathrm{E}-02$ & \\
\hline STR & $\begin{array}{l}\text { Scaled register } \\
\text { offset }\end{array}$ & $7.27 \mathrm{E}-03$ & \\
\hline
\end{tabular}

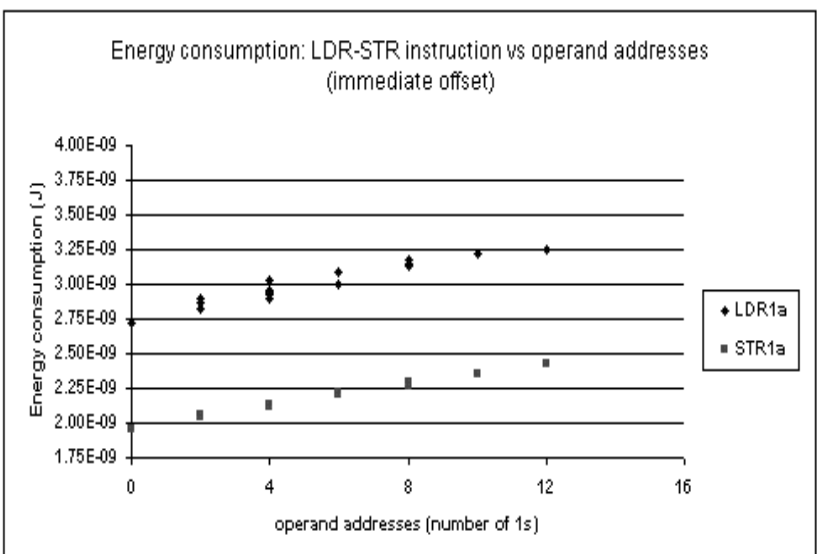

Fig. 4. Energy consumption versus the number of $1 \mathrm{~s}$ in the operand addresses for the LDR and STR instructions (immediate offset) 


\section{ANALYSIS OF THE EFFECT OF PIPELINE STALLS AND FLUSHES}

The ARM processor supports the RISC architectural paradigm. Moreover, the processing of the ARM instructions is subdivided into three pipeline stages (Instruction Fetch Instruction Decode - Execute) to improve performance in terms of throughput.

Generally, the processing flow can be delayed due to a condition that cannot be satisfied at the time needed. When such a situation occurs, the pipeline is stalled and this irregularity results in a pipeline stage with no useful computations performed. In the case of the ARM processor core, we distinguish two categories of such conditions, a) pipeline flushes due to altering the control flow of the program by introducing a non-sequential value to the $\mathrm{PC}$ and b) pipeline stalls when the condition defined for a conditionally executed instruction is not satisfied.

For these types of pipeline stalls, small program loops to activate corresponding conditions have been implemented in order to measure their effect on the energy consumption. For each type of these stall cases in the pipeline, it is found that a single absolute overhead to describe their cost in energy is sufficient. The corresponding overhead values are shown in Table 8
Table VIII. Model parameters for the effect of pipeline stalls

\begin{tabular}{|l|c|}
\hline Instruction type & Absolute overhead to base cost (nJoules) \\
\hline Pipeline flush & 2.04 \\
\hline Any & \\
\hline Pipeline stalls \\
\hline $\begin{array}{l}\text { Data processing } \\
\text { and load-store }\end{array}$ \\
\hline Multiply & 1.08 \\
\hline Branch & 1.46 \\
\hline
\end{tabular}

To evaluate the absolute accuracy of our modeling approach, real programs were used as benchmarks. Table 9 gives the measured and estimated energy consumption for a small program kernel. The corresponding assembly list has been extracted from a $\mathrm{C}$ program by utilizing the facilities of the armcc tool, shipped with the ARM ADS software distribution. The measured values correspond to the energy consumption during the cycles while the estimated values correspond to the consumption of the instructions. The overall energy dissipation is calculated by summing up all the individual contributions that related to variations in the energy consumption at the instruction level. According to our results the error of our approach in real life programs was found to be less than $5 \%$.

Table IX. Comparison of estimated and measured energy consumption of a real kernel

\begin{tabular}{|c|c|c|c|c|c|c|c|c|c|}
\hline Instruction formation & Cycle & Measured & Ebase & Einter & Eregnum & Eregval & Eimm & Estall & Estimated \\
\hline MOV a1, \#5 & 1 & $9.248 \mathrm{E}-01$ & 0.930 & & \begin{tabular}{|l|}
0.000 \\
\end{tabular} & \begin{tabular}{|l|}
0.000 \\
\end{tabular} & 0.069 & & $\begin{array}{l}0.999 \\
\end{array}$ \\
\hline SUB a1, a1, \#1 & 2 & 9.431E-01 & 0.800 & -0.092 & 0.000 & 0.015 & 0.068 & & 0.791 \\
\hline CMP a1, \#1 & 3 & $8.109 \mathrm{E}-01$ & 0.830 & -0.112 & 0.000 & 0.008 & 0.068 & & 0.793 \\
\hline \multirow[t]{3}{*}{ BGT label } & 4 & $7.680 \mathrm{E}-01$ & 3.100 & -0.130 & & & & & 2.970 \\
\hline & 5 & 1.022 & & -0.032 & & & & & -0.032 \\
\hline & 6 & 9.213E-01 & & & & & & & 0.000 \\
\hline SUB a1, a1, \#1 & 7 & $9.050 \mathrm{E}-01$ & 0.800 & & 0.000 & 0.008 & 0.068 & & 0.875 \\
\hline CMP a1, \#1 & 8 & 9.503E-01 & 0.830 & -0.112 & 0.000 & 0.015 & 0.068 & & 0.801 \\
\hline \multirow[t]{3}{*}{ BGT label } & 9 & 7.671E-01 & 3.100 & -0.130 & & & & & 2.970 \\
\hline & 10 & 1.025 & & -0.032 & & & & & -0.032 \\
\hline & 11 & 9.262E-01 & & & & & & & 0.000 \\
\hline SUB a1, a1, \#1 & 12 & $9.040 \mathrm{E}-01$ & 0.800 & & 0.000 & 0.015 & 0.068 & & 0.883 \\
\hline CMP a1, \#1 & 13 & 9.442E-01 & 0.830 & -0.112 & 0.000 & 0.008 & 0.068 & & 0.793 \\
\hline \multirow[t]{3}{*}{ BGT label } & 14 & 7.679E-01 & 3.100 & -0.130 & & & & & 2.970 \\
\hline & 15 & 1.023 & & -0.032 & & & & & -0.032 \\
\hline & 16 & $9.262 \mathrm{E}-01$ & & & & & & & 0.000 \\
\hline SUB a1, a1, \#1 & 17 & $9.086 \mathrm{E}-01$ & 0.800 & & 0.000 & 0.008 & 0.068 & & 0.875 \\
\hline CMP a1, \#1 & 18 & $9.457 \mathrm{E}-01$ & 0.830 & -0.112 & 0.000 & 0.008 & 0.068 & & 0.793 \\
\hline BGT label & 19 & $7.714 \mathrm{E}-01$ & & -0.092 & & & & 1.601 & 1.509 \\
\hline \multirow[t]{3}{*}{ MOV a1, \#0 } & 20 & 1.053 & 0.930 & & 0.000 & 0.008 & 0.000 & & 0.938 \\
\hline & 21 & 1.232 & & & & & 0.000 & & 0.000 \\
\hline & 22 & $9.380 \mathrm{E}-01$ & & & & & & & 0.000 \\
\hline Total & & 18.571 & & & & & & & 18.865 \\
\hline$\%$ difference & & & & & & & & & -1.58 \\
\hline
\end{tabular}




\section{CONCLUSIONS}

The measurements taken for the development of instruction-level energy models for the ARM7TDMI embedded processor are presented and analyzed. The instantaneous current drawn by the processor is measured and integrated in clock cycles to derive the consumed energy. Appropriate measuring environment and measuring methodology has been established for this purpose. The energy of an instruction is analyzed in three components so that the proposed modeling technique can be applied. These components correspond to the pure base energy cost of the instruction, the inter-instruction cost and the effect of the instruction parameters. The values for these components were presented, analyzed and discussed. The proposed approach in modeling the software energy of microprocessors is validated by the results since only up to $5 \%$ error in energy was observed for real software kernels.

\section{ACKNOWLEDGMENT}

This work is performed within the project IST-200030093-EASY, funded by European Union's IST framework program.

\section{REFERENCES}

[1] Vivek Tiwari, Sharad Malik, and Andrew Wolfe, "Power Analysis of Embedded Software: A First Step Towards Software Power Minimization," IEEE Transaction on Very Large Scale Integration (VLSI) Systems, Vol. 2, No. 4, pp. 437-445, December 1994.

[2] Vivek Tiwari, Sharad Malik, Andrew Wolfe, and Mike Tien-Chen Lee, "Instruction Level Power Analysis and Optimization of Software," Journal of VLSI Signal Processing, Vol. 13, No. 2-3, pp. 223-238, August 1996.

[3] Mike Tien-Chen Lee, Vivek Tiwari, Sharad Malik, and Masahiro Fujita, "Power Analysis and Minimization Tehniques for Embedded DSP Software," IEEE Transactions on Very Large Scale Integration (VLSI) Systems, pp. 123-135, March 1997.

[4] V. Tiwari and T.C. Lee, "Power Analysis of a 32-bit Embedded Microcontroller," VLSI Design Journal, Vol. 7, No. 3, 1998.

[5] S. Steinke, M. Knauer, L. Wehmeyer, and P. Marwedel, "An Accurate and Fine Grain Instruction-Level Energy Model supporting Software Optimizations," in Proceedings of the International Workshop PATMOS, Yverdon-les-bains, Switzerland, September 2001.

[6] J. T. Russell, and M. F. Jacome, "Software Power Estimation and Optimization for High Performance, 32-bit Embedded Processors," in Proceedings of the International Conference on Computer Design (ICCD '98), October 1998.

[7] Naehyuck Chang, Kwanho Kim, and Hyun Gyu Lee, "Cycle-Accurate Energy Consumption Measurement and Analysis: Case Study of ARM7TDMI," IEEE Transactions on VLSI Systems, Vol. 10, No. 2, pp. 146-154, April 2002.

[8] T. Laopoulos, P. Neofotistos, K. Kosmatopoulos, and S. Nikolaidis, "Measurement of Current Variations for the Estimation of Softwarerelated Power Consumption," accepted in IEEE Trans. on Instrumentation and Measurement.

[9] S. Nikolaidis, N. Kavvadias, P. Neofotistos, K. Kosmatopoulos, T. Laopoulos, and L. Bisdounis, "Instrumentation set-up for Instruction Level Power Modeling," Int. Workshop on Power and Timing Modeling, Optimization and Simulation, Seville, Spain, Sept. 2002
[10] S. Nikolaidis, N. Kavvadias, and P. Neofotistos, "Instruction level power measurements and analysis", IST-2000-30093/EASY Project, Deliverable 15, Sept 2002. http://easy.intranet.gr

[11] S. Nikolaidis, N. Kavvadias, and P. Neofotistos, "Instruction level power models for embedded processors", IST-2000-30093/EASY Project, Deliverable 21, Dec 2002. http://easy.intranet.gr 